\title{
A New Method for the Calculation of Propagation Constants and Field Profiles of Guided Modes of Nonlinear Channel Waveguides Based on the Effective Index Method
}

Gijs J. M. Krijnen, Member, IEEE, Hugo J. W. M. Hoekstra, and Paul V. Lambeck

\begin{abstract}
In this paper, an extension of the effective index method (EIM) to waveguiding structures containing ideal or saturable third-order nonlinear materials is presented. By applying separation of variables to the dominant field component, the complete problem is subdivided into two scalar problems in the lateral and transverse direction, as in the case of the normal EIM. Making use of the strong transverse confinement, as observed in most real waveguide structures, the nonlinear index changes of the various transverse sections can be lumped into nonlinear effective indexes of the equivalent layered planar structures. By using these nonlinear effective indexes in selfconsistent field calculations in the transverse direction, a complete approximate solution is obtained. In this way, the amount of computational effort required for the calculation of the effective indexes and field profiles of the waveguides can be reduced significantly.
\end{abstract}

\section{INTRODUCTION}

$\mathrm{T}$ HE effective index method (EIM) is a well-known and widely used method for the calculation of propagation constants and field profiles of guided modes of linear channel waveguides. It consists of approximating the electric (quasi-TE) or magnetic (quasi-TM) fields by their dominant components ( $E_{y}$ or $H_{y}$ ), and subsequently separating the $x$ and $y$ dependence of these components (see Fig. 1), thereby transforming the complete vectorial problem into two scalar, one-dimensional, and easy to solve problems [1]. Although the method is only approximate, it has been shown (see, for example, [2]-[4]) that it can produce accurate results provided that the modes

Manuscript received August 18, 1993; revised March 14, 1994. The work of G. J. M. Krijnen was supported by a Fellowship from the Royal Netherlands Academy of Arts and Sciences.

G. J. M. Krijnen is with the Lightwave Device Group, MESA Research Institute, University of Twente, 7500AE, Enschede, The Netherlands, on leave at CREOL, University of Central Florida, Orlando, FL 32826 .

H. J. W. M. Hoekstra and P. V. Lambeck are with the Lightwave Device Group, MESA Research Institute, University of Twente, 7500AE, Enschede, The Netherlands.

IEEE Log Number 9402771.

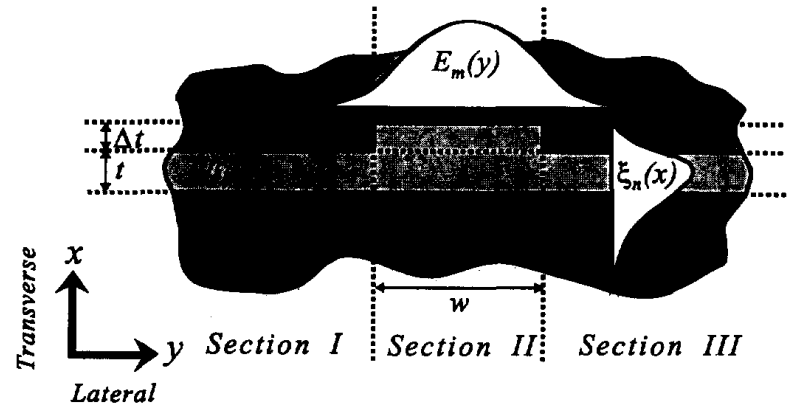

Fig. 1. Schematic of the considered structures, definition of the coordinate system, and illustration of the two functions as used by separation of variables.

considered are far from cutoff, that they have an elongated mode profile, and that they show only small variations in at least one direction. Since integrated optics structures are often produced by planar technologies (such as thin-film deposition and etching) with only small variations of the refractive index in the lateral direction, this situation is frequently met, making the EIM a very useful tool for design purposes. In the case where structures comprising Kerr-like nonlinearities, i.e.,

$$
n=n_{0}+n_{2 E}|E|^{2}
$$

are considered, the situation becomes more complicated. Although there are many analytical and numerical methods by which nonlinear slab waveguides can be analyzed [5]-[10], the problem becomes even more complex when channel waveguides are concerned. For nonlinear waveguides, two different regimes can be distinguished [11]; either the nonlinearity is small compared to the linear index contrast (i.e., $n_{2 E}|E|^{2} / \Delta n_{0} \ll 1$ with $\Delta n_{0}$ the linear index contrast) and the field profiles are only slightly power dependent, or the nonlinear induced index chang- 
es are large compared to the index contrast (i.e., $\left.n_{2 E}|E|^{2} / \Delta n_{0} \geq 1\right)$ and the field profiles depend strongly on the guided mode power. In the first case, the power dependence of the mode propagation constants can be calculated using the nonlinear overlap integrals as given by coupled mode analysis (CMA) [12]. In the second case, this approach is not allowed since the assumption that the modal fields do not change much as a function of the guided mode power (which is inherent in the CMA) is no longer valid. A combination of $n_{2 E}|E|^{2} / \Delta n_{0} \ll 1$ and $n_{2 E}|E|^{2} / \Delta N_{0} \geq 1$ is found in many waveguide structures where the light is well confined in the transverse direction (normal to the substrate) for which $\Delta n_{0}$ is typically quite high $\left(\Delta n_{0} \approx 0.05-2\right)$, but only loosely confined in the lateral direction where a small effective index contrast $\left(\Delta N_{0} \approx 0.001-0.01\right)$ is used. This suggests that, although the transverse field shape is hardly affected by nonlinear induced index changes (since these are typically $\varangle 0.05$ ), the lateral field shape may strongly depend on the guided mode power due to the small lateral effective index contrast. Thus, both the shape and the magnitude of the field distribution are important now.

The most rigorous methods to solve for the modes of nonlinear channel waveguides are numerical methods based on self-consistency and taking the vectorial nature of the problem fully into account. However, results may depend on the specific numerical parameters (such as grid sizes used), and sometimes spurious solutions are generated (due to specific boundary constraints). The major drawback of these methods is that they generally are very time consuming and occupy large memory resources. Examples of these approaches can be found in the literature for Kerr nonlinearities [13] as well as for saturable materials [14]-[17].

In some situations, it can be advantageous to use nonlinear variants of the EIM because they: 1) are generally relatively simple, 2) give much insight, and 3) require little CPU time. Several publications dealing with simplified analyses of two-dimensional nonlinear waveguiding problems have emerged in the past. In [18], rib waveguides on nonlinear substrates were analyzed by using a numerical approach based on the EIM in combination with the use of the WKB method [19]. This approach requires the use of specific functions depending on the geometry of the waveguide and the order of the mode for which to find a solution. A method based on separation of variables in combination with the use of variational expressions was introduced in [20] and compared to numerical results in [17] showing good correspondence. Moreover, in [21], it was shown that for the case of spatial solitons in planar waveguides with even a small linear refractive index difference of 0.02 , the assumption of separable variables was met very well when compared to full vectorial numerical solutions. In [22], a nonlinear extension of the EIM was introduced and applied to the end of iteratively calculating the field profiles and propagation constants of spatial solitons propagating in planar waveguides containing Kerr nonlinearities.
In this paper, an extension of the EIM to a nonlinear effective index method (NEIM), applicable to a restricted but large group of channel nonlinear waveguide structures, will be introduced. The waveguide may contain ideal or saturable nonlinear materials, and the method can be applied to arbitrary mode orders provided that they exist in the linear limit. Intermediate results produced by the method have the form of effective nonlinear indexes, and can be used in the complete calculations of the modes, but also in other two-dimensional calculations like $2 \mathrm{D}$ beam propagation method calculations, thus enabling the analysis of $z$-invariant structures with modest means. An example of the application of the method will be given.

\section{Nonlinear EfFective Index Method}

The general formulation of the problem is given by the nonlinear wave equation

$$
\nabla \boldsymbol{E}(\boldsymbol{r})+k_{0}^{2}\left[n_{0}(\boldsymbol{r})+\Delta n\left(|\boldsymbol{E}(\boldsymbol{r})|^{2}\right)\right]^{2} \boldsymbol{E}(\boldsymbol{r})=0
$$

where the time dependence $e^{j \omega t}$ is implicit, $\boldsymbol{E}(\boldsymbol{r})$ is the electrical field, $k_{0}$ is the free-space wavenumber $(\omega / c)$, and $\Delta n\left(|E(r)|^{2}\right)$ is the nonlinear induced index change (e.g., $n_{2 E}|\boldsymbol{E}(\boldsymbol{r})|^{2}$ in the case of ideal nonlinear media). For straight waveguides (i.e., structures that do not depend on $z$ ), stable modal solutions can be described by

$$
\boldsymbol{E}(\boldsymbol{r})=\boldsymbol{E}_{n m}(x, y) e^{-j \beta z}
$$

where $\beta$ is the propagation constant, $\boldsymbol{E}_{n m}(x, y)$ is the field profile of the guided mode, and $n$ and $m$ are the lateral and transversal mode numbers, respectively. In nonlinear structures, both $\beta$ and $\boldsymbol{E}_{n m}(x, y)$ will, in general, depend on the guided mode power, and it is the purpose of the NEIM to find approximate solutions for this power dependence.

\section{A. Effective Nonlinear Indexes}

As in the normal EIM, the starting point for the NEIM is the approximation of the complete vectorial field $\left(E_{n m}(x, y)\right)$ by its dominant field component only $\left(E_{y}, H_{y}\right.$ for quasi-TE and quasi-TM polarization, respectively), and subsequently separating the $x$ and $y$ dependence of these components, i.e., writing down the complete field as a product of two functions $A_{x}(x) A_{y}(y)$. However, this is only allowed when the considered structures comply with a few requirements, thus limiting the applicability of the method to a restricted but large class of waveguiding structures. 1) The first requirement is that the structures can be subdivided into multiple transverse sections for which the effective indexes and field profiles can be calculated for the equivalent slab waveguides. 2) Second, it is necessary that the mode profiles have an elongated shape which can be obtained, for example, by a waveguide width $(w)$ to waveguide thickness $(t)$ ratio far from one (e.g., $w / t \ll 1$ or $w / t \gg 1$ ). 3) Third, there may only be small differences between the modes of the slab waveguide equivalents of the different transverse sections (e.g., $\left(n_{f}-\right.$ $\left.n_{c}\right)^{*} \Delta t<t n_{f}$; see Fig. 1). 4) Finally, it is necessary that the 
mutual refractive index difference $\left(\Delta n_{0}\right)$ of all layers is much larger than the maximum obtainable nonlinear index change.

Note that requirement 1 ) is also a necessary condition when dealing with linear structures if the complete field profiles have to be calculated (although it is not necessary for the calculation of the effective indexes). Requirements 2) and 3) are also found in the normal EIM, and stem from the fact that the fields must have one dominant vector component [requirement 2 )] and a nearly continuous shape in at least one direction in order to allow for separation of variables [requirement 3)]. So the only extra condition imposed by our nonlinear extension of the EIM is 4). But it is by virtue of this condition that the field distributions in the transverse direction are hardly dependent on the guided mode power. This enables us to describe the nonlinear effects solely by power-dependent effective mode indexes which can be used subsequently in the calculations in the lateral direction.

In the following, a derivation of the method will be given for quasi-TE ${ }_{n m}$ modes, although, of course, the method can also be applied to quasi-TM ${ }_{n m}$ modes. The coordinate system used is given in Fig. 1. If the conditions given above are fulfilled, the field profile of a quasi-TE ${ }_{n m}$ mode, propagating in the positive $z$ direction, may be approximated, after separation of variables, by

$$
E_{n m}(x, y, z, P)=C \xi_{n}(x) E_{m}(y, P) e^{-j \beta_{n m}(P) z}
$$

where $C$ is a dimensionless quantity having a magnitude equal to the square root of the guided mode power $(\sqrt{P})$ and $\beta_{n m}$ is the power-dependent propagation constant. In order to apply the NEIM, it is important to take care of the total power that is carried by the modes. In order to have the guided mode power calculated properly, $\xi_{n}$ is a dimensionless function representing the powerindependent field profile in the transverse $(x)$ direction with normalization to 1 , i.e.,

$$
\int_{-\infty}^{+\infty}\left|\xi_{n}\right|^{2} d x=1 \mathrm{~m}
$$

whereas $E_{m}(y, P)$ represents the power-dependent field profile in the lateral $(y)$ direction normalized to $1 \mathrm{~W} / \mathrm{m}$ (so $E_{m}$ has the dimension $\mathrm{V} / \mathrm{m}$ ) and

$$
\frac{\beta_{n m}}{2 \omega \mu_{0}} \int_{-\infty}^{+\infty}\left|E_{m}(y, P)\right|^{2} d y=1 \mathrm{~W} / \mathrm{m}
$$

Note that the total guided mode power $P$ is given by

$$
P=C^{2} \frac{\beta_{n m}}{2 \omega \mu_{0}} \int_{-\infty}^{+\infty}\left|E_{m}(y, P)\right|^{2} d y \int_{-\infty}^{+\infty}\left|\xi_{n}\right|^{2} d x=C^{2} \mathrm{~W} .
$$

The power per unit of length along the $y$ axis $P_{y}(y, P)$, on the other hand, is given by

$$
\begin{aligned}
P_{y}(y, P) & =C^{2} \frac{\beta_{n m}}{2 \omega \mu_{0}} \int_{-\infty}^{+\infty}\left|E_{m}(y, P)\right|^{2}\left|\xi_{n}\right|^{2} d x \\
& =C^{2} \frac{\beta_{n m}}{2 \omega \mu_{0}}\left|E_{m}(y, P)\right|^{2} \mathrm{~W} / \mathrm{m} .
\end{aligned}
$$

The determination of the complete mode profile starts with the calculation of the power-independent transverse field profile $\left(\xi_{n}\right)$. By using a slab solver (e.g., making use of transfer matrices and solving for the propagation constants [9]), the effective indexes and field profiles of the modes in the transverse sections (e.g., sections I, II, and III in Fig. 1) are found. Next, the power dependence of the effective indexes in these transverse sections is obtained from the nonlinear overlap integrals as given by coupled mode analysis [12]:

$$
\begin{gathered}
\left.N_{n}^{i}\left(y, P_{y}\right) \approx N_{n}^{i}\right|_{P_{y}=0}+\frac{Q_{n}^{i}}{k_{0}} P_{y}(y, P) \\
Q_{n}^{i}=\frac{\omega \epsilon_{0}}{2} \int_{-\infty}^{+\infty} n_{0}(x) n_{2 E}(x)\left|E_{n}^{i}(x)\right|^{4} d x \mathrm{~W}^{-1}
\end{gathered}
$$

where $N_{n}^{i}, Q_{n}^{i}$, and $E_{n}^{i}$ denote, respectively, the effective index, the nonlinear coefficient of self-modulation, and the transverse mode profile of the $n$ th-order mode in the $i$ th transverse section. Note that $E_{n}^{i}$ is normalized to 1 W/m (i.e., $\left.E_{n}^{i}=\xi_{n}^{i}(x) \sqrt{2 \omega \mu_{0} / \beta_{n}^{i}}\right)$. The next objective is to find an effective nonlinear index that can be applied in calculations in the lateral direction in order to determine the power dependence of the guided modes of the channel waveguide (or alternatively, as an input for twodimensional propagation algorithms for $z$-variant structures). On substituting (8) into (9a), one obtains

$$
\left.N_{n}^{i}\left(y, P_{y}\right) \approx N_{n}^{i}\right|_{P_{y}=0}+\frac{Q_{n}^{i}}{k_{0}} \frac{\beta_{n}^{i}}{2 \omega \mu_{0}}\left|C E_{m}(y)\right|^{2} .
$$

Here, $\beta_{n m}$ has been replaced by $\beta_{n}^{i}$, which is allowed whenever the EIM may be applied since, typically, the difference between both values is very small $(<1 \%)$. So the new effective nonlinear index, to be used in the calculations in the lateral direction, is given by

$$
N_{2 E n}^{i}=\frac{Q_{n}^{i}}{k_{0}} \frac{\beta_{n}^{i}}{2 \omega \mu_{0}}=\frac{Q_{n}^{i} N_{n}^{i}}{2 \omega \mu_{0}} .
$$

On inserting (4) and (11) into (2), the resulting equation to be solved becomes

$$
\begin{array}{r}
\frac{\partial^{2} E_{m}(y, P)}{\partial y^{2}}+\left\{k _ { 0 } ^ { 2 } \left[N_{n}(y)\right.\right. \\
\left.+\Delta N_{n}\left(y,\left|C E_{m}(y, P)\right|^{2}\right)\right]^{2} \\
\left.-\beta_{n m}^{2}(P)\right\} E_{m}(y, P)=0
\end{array}
$$

where $\Delta N_{n}$ is a function for the effective nonlinear index change (which is equal to $N_{2 E, n}^{i}\left|C E_{m}(y, P)\right|^{2}$ for Kerr nonlinearities).

\section{B. Method of Self-Consistent Fields}

Since the nonlinear induced effective index changes can be larger than the linear effective index contrasts, the lateral field profiles may be strongly power dependent. So it is not allowed to use CMA [i.e., (9)] for the calculations 
in this direction again. Instead, we use a numerical method (here referred to as the method of self-consistent fields (MSCF) [10], [23], [24]) in order to calculate the field profiles and effective indexes of nonlinear slab waveguides at given input powers. The method is applicable to guided modes that exist in the linear limit, and can handle the nonlinear induced (effective) index changes normally encountered in real nonlinear materials easily. The algorithm is schematically depicted in Fig. 2. In the first step, the field profile and effective index are calculated in the $P \rightarrow 0$ limit using a standard slab solver. In the second step, the original number of $k$ "physical" layers of the structure is expanded to a much larger number (typically $\approx 160$ ) of $l$ "numerical" layers. This is done adaptively in such a way that the density of layers is large at those positions where large nonlinear index changes can be expected (i.e., where the product of intensity and nonlinear index is high). By taking a large number of layers, the thickness of each "numerical" layer becomes small, thus allowing for an accurate approximation of the index profile by a staircase curve. Since the method is used to calculate modes that also exist in the linear limit, the field profiles are exponentially decaying in the outer layers. Therefore, the number of numerical layers in the physical outer layers is limited, and the two most outer numerical layers ( 1 and $l$ ) far from the core of the waveguide can be taken linear. Fig. 7 shows an example of the staircase curves as obtained by the adaptive procedure. In the third step, the nonlinearity is taken into account by calculating the induced index changes from the mode profile and the nonlinear materials response [e.g., according to (1) or (13)]. The (effective) index structure thus obtained is regarded as a linear structure, and its propagation constant and field profiles are obtained using a standard slab solver again. Subsequently, the calculations are iterated from the third step until the index changes between two successive iteration steps $\left(\delta n_{1} \cdots \delta n_{l}\right)$ are smaller than a prespecified lower limit $\Delta n\left(1 \cdot 10^{-8}\right.$ in the implementation used here) for each layer. At this point, the index profile and the field profile are correctly related, i.e., they are consistent.

The validity and the accuracy of the method were verified [24] against analytical solutions as given in [7] for a structure as described in the same reference. It appeared that the results were indistinguishable for nonlinear index increments of up to 0.5 , showing some deviations at higher values. However, such high nonlinear index changes are hardly ever met (except for liquid crystals), and thus, we can conclude that the MSCF can be applied safely for a large class of nonlinear slab waveguides.

\section{Inclusion of Saturable Nonlinear Indexes in the NEIM}

An interesting aspect of the MSCF is that it allows for the modeling of materials with saturable nonlinear indexes. Since, in the MSCF, in each iteration the local nonlinear index changes are calculated from the local intensity and the nonlinearity, it is straightforward to include a specific nonlinear materials response. For the

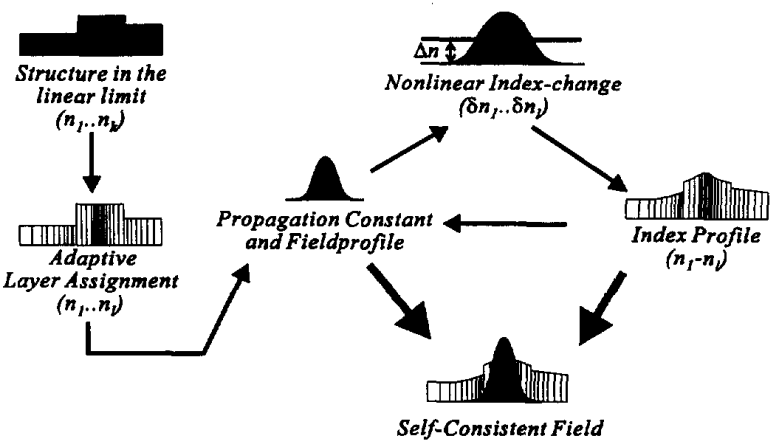

Fig. 2. Schematic representation of the method of self-consistent fields (MSCF) (see text).

calculations including saturation as presented below, we used either an exponential saturable nonlinearity given by [14]

$$
\Delta n(E)=\Delta n_{\text {sat }}\left(1-e^{-n_{2 E}|E|^{2} / \Delta n_{\text {sat }}}\right)
$$

or a two-level system with saturable nonlinearity given by [25]

$$
\Delta n(E)=\frac{\Delta n_{\mathrm{sat}} \cdot n_{2 E}|E|^{2}}{\Delta n_{\mathrm{sat}}+n_{2 E}|E|^{2}} .
$$

Since our goal is to use the NEIM for the analysis of nonlinear channel waveguides, the influence of the saturation on the effective nonlinear indexes has to be determined. A proper way of including saturation effects in the NEIM is to apply the MSCF to all transverse sections and to tabulate (i.e., store in an array) the effective indexes as a function of the guided mode power. Subsequently, these tables can be used in the MSCF calculations in the lateral direction where they are applied in the same way as (13) in the transverse direction.

Alternatively a simpler, although less accurate, method can be applied. At low guided mode power, saturation is not yet significant. So the slope of the induced effective index at low power is given by the nonlinear effective index as given by (11). On the other hand, at high power, the induced effective index change will show saturation too, despite the spatial variations in intensity. In Appendix A, it is shown that the effective nonlinear index change shows a saturation behavior which is comparable to the nonlinear materials response itself. Therefore, the nonlinear effective index changes of modes of a structure containing materials with a response as given by (13) can be described approximately by

$$
\Delta N_{n}^{i}\left(y, P_{y}\right)=\Delta N_{\mathrm{sat}, n}^{i}\left(1-e^{\left.-P_{y}(y) Q_{n}^{i} / k_{0} \Delta N_{\mathrm{sat}, n}^{I}\right)}\right.
$$

for exponential saturation or by

$$
\Delta N_{n}^{i}\left(y, P_{y}\right)=\frac{\Delta N_{\mathrm{sat}, n}^{i} \cdot \frac{P_{y}(y) Q_{n}^{i}}{k_{0}}}{\Delta N_{\mathrm{sat}, n}^{i}+\frac{P_{y}(y) Q_{n}^{i}}{k_{0}}}
$$


for a nonlinearity arising from a two-level system. Comparisons of (14a) and (14b) with results of MSCF calculations are shown for $\mathrm{a} \mathrm{TE}_{0}$ and $\mathrm{TE}_{1}$ mode in the upper and lower graphs of Fig. 3, respectively. It shows that expressions (14a) and (14b) are very reasonable approximations of the induced effective index changes. Therefore, when using (14a) or (14b), it is sufficient to apply MSCF calculations in the lateral direction only.

\section{RESULTS}

In order to illustrate the use of the NEIM, it is applied to a structure as depicted in Fig. 1 with parameters as summarized in Table I. It consists of a channel waveguide formed by a film layer of $1 \mu \mathrm{m}(t)$ thickness and a refractive index $\left(n_{f}\right)$ of 1.6 on a substrate with index $\left(n_{s}\right)$ 1.5. The cladding is assumed to be air. The core of the channel waveguide is formed by reduction of the film thickness $(\Delta t)$ in Sections I and III by $0.0744 \mu \mathrm{m}$. The width of the channel $(w)$ is taken as $4 \mu \mathrm{m}$. It is assumed that the film and the substrate have a nonlinear index $n_{2 E}=2 \cdot 10^{-19} \mathrm{~m}^{2} / \mathrm{V}^{2}$, which is roughly comparable to the values found for some polymers [26]. Two types of calculations, namely, with ideal nonlinearities and with saturable nonlinearities, are performed. In the latter, $\Delta n_{\text {sat }}$ is set at 0.01 . The free-space wavelength $\lambda_{0}$ is set at 1.320 $\mu \mathrm{m}$. Calculation of the effective indexes and overlap integrals (9b) yields $N_{0}^{\mathrm{I}}=N_{0}^{\mathrm{III}}=1.540682, Q_{00}^{\mathrm{I}}=Q_{00}^{\mathrm{III}}=$ $3.909 \cdot 10^{-9} \mathrm{~m} / \mathrm{W}$ and $N_{0}^{\mathrm{II}}=1.545682, Q_{00}^{\mathrm{II}}=3.849$. $10^{-9} \mathrm{~m} / \mathrm{W}$. With these values, the effective index of the quasi $\mathrm{TE}_{00}$ channel mode in the linear limit becomes 1.543312. In order to obtain the effective nonlinear indexes to be used in the NEIM, (11) is applied. This yields $N_{2 E 0}^{\mathrm{I}}=N_{2 E 0}^{\mathrm{III}}=1.679 \cdot 10^{-13} \mathrm{~m}^{2} / \mathrm{V}^{2}$ and $N_{2 E 0}^{\mathrm{II}}=1.659$. $10^{-13} \mathrm{~m}^{2} / \mathrm{V}^{2}$.

Fig. 3 (upper graph) shows MSCF calculations of the fundamental TE mode of the equivalent slab waveguide of transverse section II. It shows the changes of the effective index as a function of guided mode power $(0 \cdots 500 \mathrm{MW} / \mathrm{m}$, the upper value roughly corresponding to a guided mode power of $2 \mathrm{~kW}$ in a $4 \mu \mathrm{m}$ wide channel waveguide). For the case of ideal nonlinear materials (no saturation), the CMA expressions for the effective index change (9) give a very reasonable approximation of the more elaborate MSCF calculations despite the fact that the maximum index changes amount to values as large as 0.063 at $P=500 \mathrm{MW} / \mathrm{m}$. For the case of saturable materials, (14) is seen to give a satisfying approximation. At high power, the effective index changes saturate at $\approx 0.01$. So, in subsequent calculations, $\Delta N_{\text {sat }}$ is taken as 0.01 too. Note that the effective linear index contrast $\left(\Delta N_{0}\right)$ is only 0.005 , and thus, smaller than $\Delta N_{\text {sat }}$. Therefore, it can be expected that the lateral field profile shows a strong power dependence, even in the case of a saturable nonlinearity of 0.01 .

As stated before, it is assumed that the field profiles are power-independent in the transverse direction. Fig. 4, displaying MSCF calculations of these transverse field profiles for the case of ideal nonlinearities, shows that this
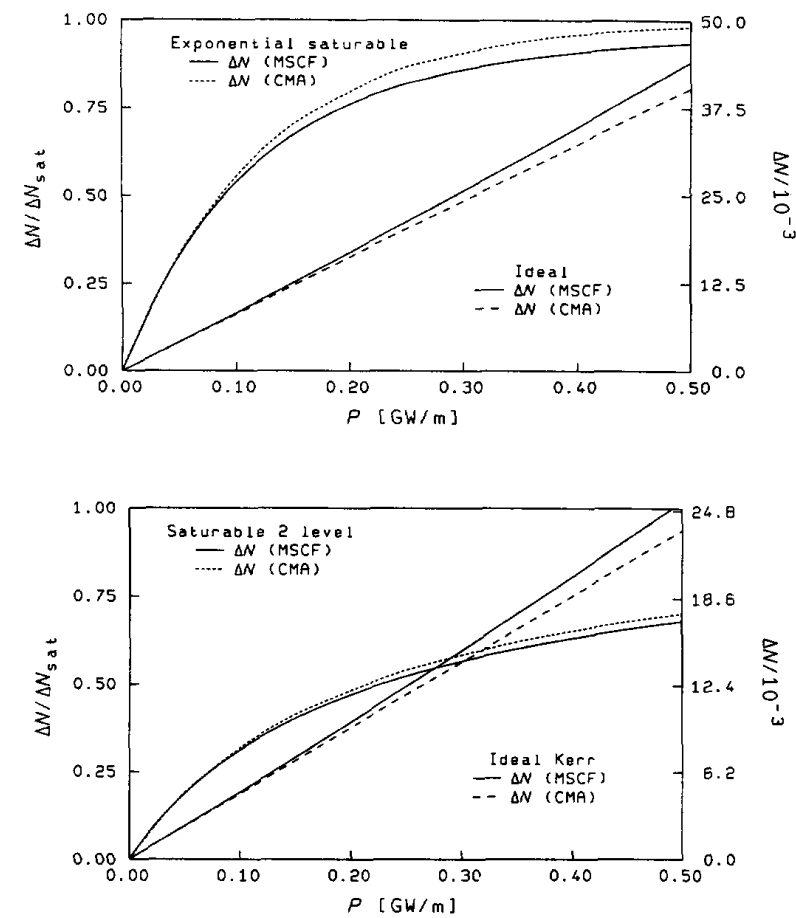

Fig. 3. Effective index change versus input power. The solid curves were calculated by means of the MSCF. The dashed lines are calculated by taking the overlap integrals (as given by CMA) and using these in the approximate expressions (14). The upper graph shows the power dependence of the $\mathrm{TE}_{0}$ mode of section II of Table I assuming exponential saturable nonlinearities. The lower graph shows the power dependence of the $\mathrm{TE}_{1}$ mode of a structure similar to section II given in Table I, but with a film thickness of $2 \mu \mathrm{m}$ and with two-level saturable nonlinearities.

is indeed the case. Although the field profiles show some variations with increasing input power, one must be aware of the fact that the accompanying nonlinear index changes are much larger $(\approx 0.01 \cdots 0.07)$ than usually found in real materials. In case saturation is included (not shown), the variations are even smaller. This is not unexpected since the nonlinear saturation is much smaller than the linear index contrast $\left(\Delta n_{\text {sat }} / \Delta n_{0} \leq 0.1\right)$, a condition that holds in most practical situations.

In order to obtain the lateral field profiles, MSCF calculations are subsequently applied for the $y$ direction. Fig. 5 (upper graph) shows the field profiles as a function of increasing input power $(0-2 \mathrm{~kW})$ for the case of an ideal nonlinearity. The figure shows that the field is extremely power-dependent and tends to localize in the center of the waveguide structure, i.e., at the position where the intensity is highest. However, it must be remarked that the nonlinear effective index changes accompanying these field profiles are as high as 0.16 , i.e., much larger than found in most real materials. Therefore, it seems more realistic to include saturation. MSCF calculations of the field profiles of the same structure, but now assuming exponential saturable nonlinear effective index changes (14a), are displayed in Fig. 5 (lower graph). Despite the saturation, the fields are still appreciably power- 
TABLE 1

Details of the Structure as Used in the Calculations. EFFective IndeXes,

Effective Nonlinear IndeXes, and Effective Nonlinear Saturation Apply to the TE 0 Mode of the Equivalent Slab WAVEGUIDES OF REGIONS I, II, AND III.

\begin{tabular}{|c|c|c|c|c|c|c|c|c|c|c|c|}
\hline \multirow[t]{2}{*}{ Section } & \multirow{2}{*}{$\frac{\text { Cladding }}{n_{r}}$} & \multicolumn{4}{|c|}{ Film } & \multicolumn{3}{|c|}{ Substrate } & \multicolumn{3}{|c|}{ Effective values } \\
\hline & & $n_{f}$ & $\begin{array}{c}n_{2 F} \\
\left(m^{2} / v^{2}\right)\end{array}$ & $\Delta n_{\text {sat }}$ & $\begin{array}{c}t \\
(\mu \mathrm{m})\end{array}$ & $n_{s}$ & $\begin{array}{c}n_{2 \mathrm{~F}} \\
\left(\mathrm{~m}^{2} \mathrm{~N}^{2}\right)\end{array}$ & $\Delta n_{\text {sat }}$ & $N_{\text {off }}$ & $\begin{array}{c}N_{3 \mathrm{~F}} \\
\left(\mathrm{~m}^{2} / \mathrm{N}^{2}\right)\end{array}$ & $\Delta N_{\text {sat }}$ \\
\hline I and III & 1.00 & 1.60 & $2 \cdot 10^{-19}$ & 0.01 & 0.9256 & 1.50 & $2 \cdot 10^{-19}$ & 0.01 & 1.540682 & $1.679 \cdot 10^{-13}$ & 0.01 \\
\hline II & 1.00 & 1.60 & $2 \cdot 10^{-19}$ & 0.01 & 1.0000 & 1.50 & $2 \cdot 10^{-19}$ & 0.01 & 1.545682 & $1.659 \cdot 10^{-13}$ & 0.01 \\
\hline
\end{tabular}

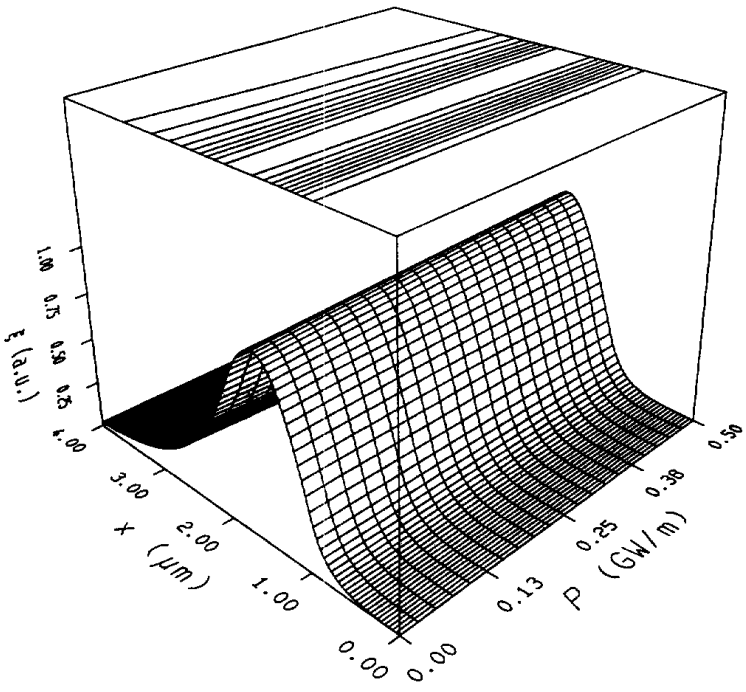

Fig. 4. Transverse field profile as a function of position and guided mode power. The input power ranges between 0 and $0.5 \mathrm{GW} / \mathrm{m}$. The structure is given in Table I with the nonlinearity assumed to be ideal (no saturation).

dependent, especially in the power interval $0-0.5 \mathrm{~kW}$ where saturation is not yet severe. At even higher values of the input power (not shown), the saturation becomes more complete, leading to relative power insensitivity and broadened fields because the linear index contrast is now controlling the field profiles again.

The complete mode profiles are obtained by combining the laterial and transverse field profiles. The thus calculated profiles are shown in Fig. 6 for input powers of 0 (upper graph), $2 \mathrm{~kW}$ and ideal nonlinearity (middle graph), and $2 \mathrm{~kW}$ and an exponential saturable nonlinearity (lower graph). A strong power dependence, clearly leading to focusing of the beam in the center of the waveguide, is demonstrated by the field profile for $P=2 \mathrm{~kW}$. However, for the case of saturable nonlinearities, the field profile for $P=2 \mathrm{~kW}$ (lower graph) is obviously more confined in the central part of the waveguide than in the linear limit, but the enhancement of the confinement is not as strong as for the ideal nonlinearity. At even higher powers (not shown), the field profiles are controlled again by the linear index contrast, which is well illustrated by the saturation
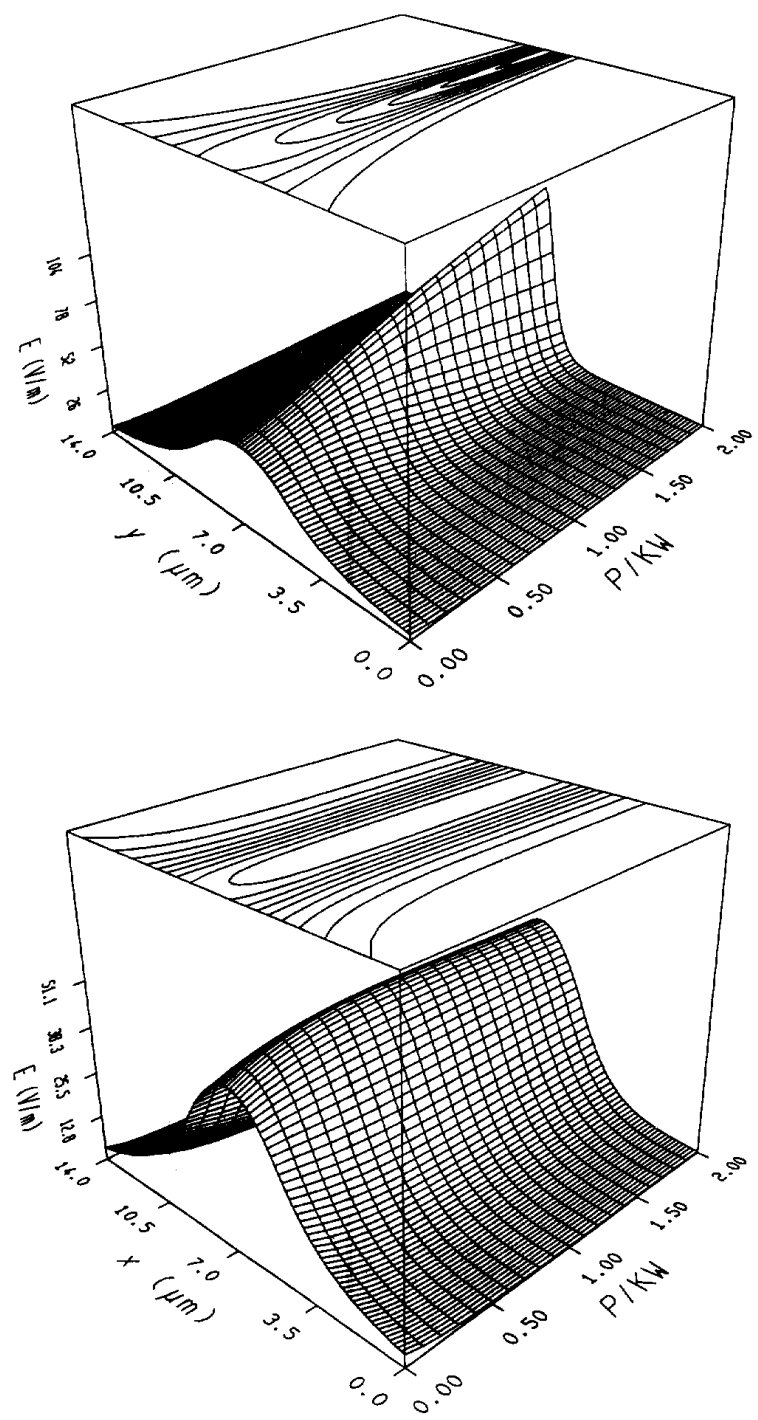

Fig. 5. Power-dependent lateral field profile of the quasi-TE $\mathbf{0 0}_{\mathbf{0}}$ mode for the sample structure (see text) as calculated by the MSCF for ideal nonlinear materials (upper graph) and saturable nonlinear materials (lower graph). 

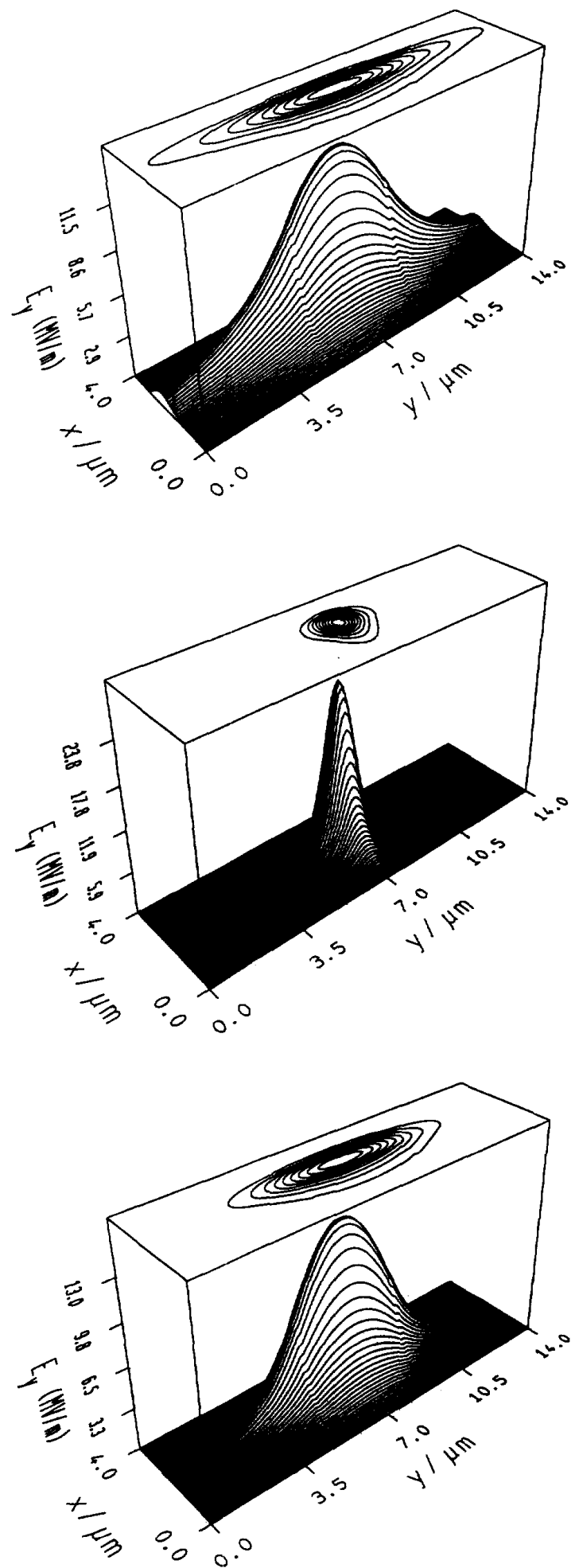

Fig. 6. Field profiles calculated according to the NEIM in the linear limit (upper graph), for $P_{\text {in }}=2 \mathrm{~kW}$ and ideal nonlinearities (middle graph), and for $P_{\text {in }}=2 \mathrm{~kW}$ and saturable nonlinearities (lower graph).

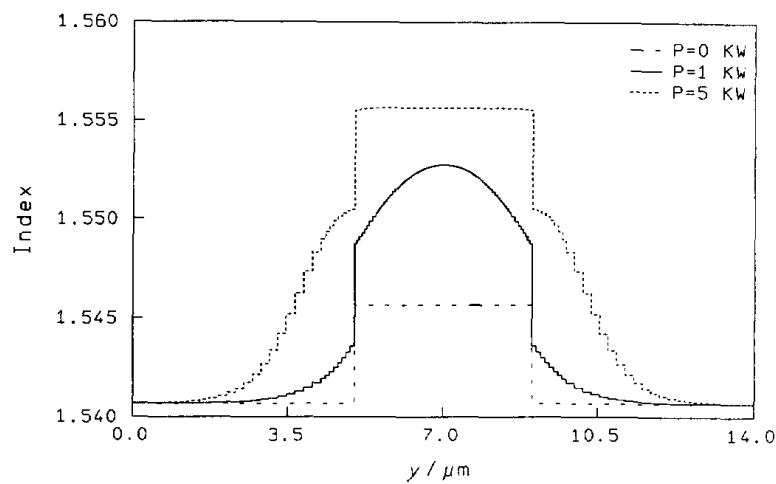

Fig. 7. Lateral effective index profiles obtained by the MSCF for input powers of 0 (dashed), 1 (solid), and 5 (dotted) $\mathrm{kW}$ for the case of exponential saturable nonlinearities.

of the lateral effective index profiles, as shown in Fig. 7 for $P=0$ (dashed line), $1 \mathrm{~kW}$ (solid line), and $5 \mathrm{~kW}$ (dotted line).

Finally, in Fig. 8, the effective index of the $\mathrm{TE}_{00}$ channel mode is plotted as a function of guided mode power. In order to roughly check the results, calculations were done in three different ways. The first method (I, solid lines) is the NEIM as presented here with the nonlinearity incorporated in the calculations by means of (11) or (14) and by using the MSCF in the lateral direction. The second method (II, dashed lines) is also the NEIM, but now by using overlap integrals ( $9 \mathrm{~b}$ ) in both directions. The third method (III, dotted lines) is derived in Appendix B, and is given by a simple expression (A.17) based on a rough approximation of the field profiles. Fig. 8 (upper graph) shows the results for the case of ideal nonlinear materials. At low power, the lines of methods I and II have the same slope, but once the power is increased to higher values, at which the MSCF calculations reveal sharpening of the lateral field profile, the slope of I increases significantly, leading to large differences at high power. At low power, the approximate expression III is seen to give results of the same order of magnitude as methods I and II, but overestimating the nonlinear effective index changes somewhat. However, at higher power, method I shows larger effective index changes than method III. The graphs for the case of saturable materials are shown in the lower part of Fig. 8. Clearly, at low power, the slopes of curves I and II are nearly equal, but at high power, when the nonlinear index starts to saturate, the two curves deviate strongly. The saturation value appears to be close to 0.01 (this is more clearly seen at even higher values of the power not shown here). Method III once again only helps to give an order of magnitude approximation at low power, but is overestimating at higher power. For low power, the nonlinear effective index change per unit of guided mode power $(\Delta N / \Delta P)$, as calculated for the $\mathrm{TE}_{00}$ channel-waveguide mode, is $12.78 \cdot 10^{-6}, 12.78$. $10^{-6}$, and $22.6 \cdot 10^{-6} \cdot \mathrm{W}^{-1}$ according to methods I, II, and III, respectively. For comparison, Fig. 8 also shows the 

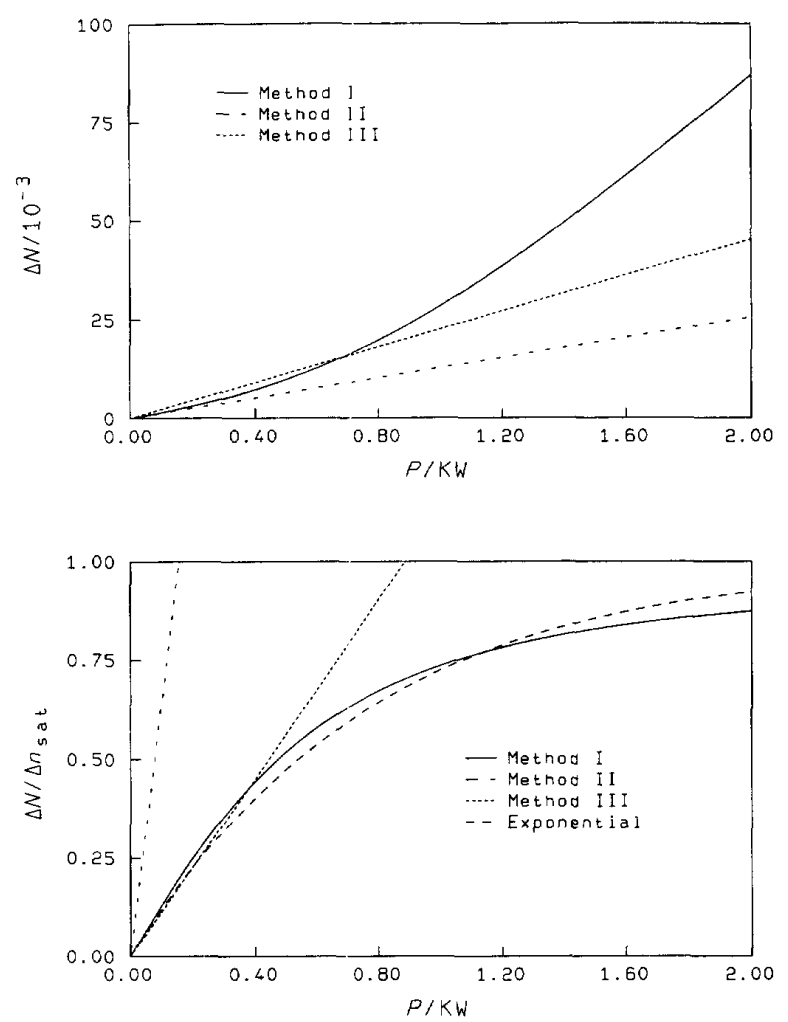

Fig. 8. Change in effective index with guided mode power as calculated by the NEIM using overlap integrals in the transverse direction and the MSCF in the lateral direction (Method I, solid), using overlap integrals in both directions (Method II, dashed), and the approximate expression equation (A.17) (Method III, dotted) for a structure with ideal nonlinearities (upper graph) and with saturable nonlinearities (lower graph). The exponential function as given by (14) (long dashes) is shown for comparison too.

exponential curve as given by (14a), with $Q_{00}$ and $\Delta N_{\text {sat }}$ taken from the calculations according to method I. It shows an interesting, good resemblance to the curve obtained with method I.

\section{CONCLUSIONS}

We have reported on an extension of the effective index method to structures comprising ideal and saturable nonlinearities. The method requires only little computing power and can be applied to a large class of waveguiding structures. In the method, the dominant field components are approximated by the product of a transverse and a lateral field profile. The method is based on the assumption that the field profiles do not change in the direction of large index contrast, whereas it allows for powerdependent field profiles in the other direction. Such power-dependent field profiles can be expected to be found in many nonlinear waveguide structures with small lateral index contrast. Results of the method were discussed on the basis of an example with realistic nonlinearities and powers. It was shown that both the power depen- dency of the field profiles as well as the nonlinear saturation cause the effective index changes to differ strongly from the results as expected from a coupled mode analysis based on power-independent field profiles and ideal nonlinear materials.

\section{APPENDIX}

\section{A. Saturation of Nonlinear Effective Indexes}

Expressions will be derived for the effective nonlinear index change of a mode propagating through a slab waveguide containing exponential saturable nonlinear materials, assuming that the field profiles do not change as a function of the guided mode power. In this case, the modal field profile of the $n$ th-order mode is written

$$
E\left(x, P_{y}\right)=\sqrt{P_{y}} E_{n}(x) e^{-j\left(N+\Delta N\left(P_{y}\right)\right) k_{0} z}
$$

where $E_{n}$ is the normalized mode profile and $P_{y}$ is the guided mode power per unit width of the waveguide. Using (13a), the induced nonlinear index changes are given by

$\Delta n\left(x, P_{y}\right)=\Delta n_{\mathrm{sat}}(x)\left(1-e^{-n_{2 E}(x) P_{y}\left|E_{n}(x)\right|^{2} / \Delta n_{\mathrm{sat}}(x)}\right)$.

Inserting (A.2) into the expression of the variation theorem [19] yields

$$
\begin{aligned}
\Delta N\left(P_{y}\right)= & \frac{\omega \epsilon_{0}}{4 k_{0}} \int_{-\infty}^{+\infty} 2 n_{0}(x) \Delta n_{\mathrm{sat}}(x) \\
& \cdot\left(1-e^{-n_{2 E}(x) P_{y}\left|E_{n}(x)\right|^{2} / \Delta n_{\mathrm{sat}}(x)}\right)\left|E_{n}(x)\right|^{2} d x .
\end{aligned}
$$

The derivative of $\Delta N\left(P_{y}\right)$ with respect to $P_{y}$ is

$$
\begin{aligned}
\frac{\partial \Delta N\left(P_{y}\right)}{\partial P_{y}}= & \frac{\omega \epsilon_{0}}{4 k_{0}} \int_{-\infty}^{+\infty} 2 n_{0}(x) n_{2 E} \\
& \cdot(x)\left|E_{n}(x)\right|^{4} e^{-n_{2 E}(x) P_{y}\left|E_{n}(x)\right|^{2} / \Delta n_{\text {sat }}(x)} d x .
\end{aligned}
$$

Taking the linear limit of (A.4), the result is

$\lim _{P_{y} \rightarrow 0} \frac{\partial \Delta N\left(P_{y}\right)}{\partial P_{y}}=\frac{\omega \epsilon_{0}}{2 k_{0}} \int_{-\infty}^{+\infty} n_{0}(x) n_{2 E}(x)\left|E_{n}(x)\right|^{4} d x \equiv \frac{Q_{n}}{k_{0}}$.

As expected, at low power, there is no difference between an ideal nonlinearity and a saturable nonlinearity. On taking the limit of $P_{y}$ to $+\infty$, on the other hand, (A.3) reduces to

$$
\begin{aligned}
\lim _{P_{y} \rightarrow \infty} \Delta N\left(P_{y}\right)= & \frac{\omega \epsilon_{0}}{2 k_{0}} \int_{-\infty}^{+\infty} n_{0} \\
& \cdot(x) \Delta n_{\text {sat }}(x)\left|E_{n}(x)\right|^{2} d x \equiv \Delta N_{\text {sat }} .
\end{aligned}
$$

When the field profile is located primarily in the layers that give the largest contributions to the induced effective index changes (or when the nonlinearity is homogeneous), it can be easily seen from (A.6) that $\Delta N_{\text {sat }} \approx \Delta n_{\text {sat }}$. In the search for a simple expression for the effective index changes, a function must be taken that complies with 
(A.5) and (A.6). With the assumptions made above, it can be expected that the effective index change as a function of the guided mode power shows a saturation behavior that is comparable to the local nonlinear materials saturation. Thus, it seems appropriate to write the effective index change as

$$
\Delta N\left(P_{y}\right)=\Delta N_{\text {sat }}\left(1-e^{-P_{y} k / \Delta N_{\text {sat }}}\right)
$$

in which $K$ is a constant. Taking the low-power limit of the derivative of (A.7) with respect to $P_{y}$ and equating this to (A.5), it turns out that $K=Q_{n} / k_{0}$. Thus, an expression for the effective index change as a function of guided mode power is given by

$$
\Delta N\left(P_{y}\right)=\Delta N_{\text {sat }}\left(1-e^{-P_{y} Q_{n} / k_{0} \Delta N_{\text {sat }}}\right) .
$$

Following the same line of reasoning, analogous relations can be obtained for two-level system-saturable nonlinearities. Replacing (A.1) by

$$
\Delta n\left(x, P_{y}\right)=\frac{\Delta n_{\text {sat }}(x) \cdot n_{2 E} P_{y}\left|E_{n}(x)\right|^{2}}{\Delta n_{\text {sat }}(x)+n_{2 E} P_{y}\left|E_{n}(x)\right|^{2}}
$$

and inserting this in the expression of the variation theorem yields

$$
\begin{aligned}
\Delta N\left(P_{y}\right)= & \frac{\omega \epsilon_{0}}{4 k_{0}} \int_{-\infty}^{+\infty} 2 n_{0}(x) \\
& \cdot \frac{\Delta n_{\mathrm{sat}}(x) \cdot n_{2 E} P_{y}\left|E_{n}(x)\right|^{2}}{\Delta n_{\mathrm{sat}}(x)+n_{2 E} P_{y}\left|E_{n}(x)\right|^{2}}\left|E_{n}(x)\right|^{2} d x .
\end{aligned}
$$

When taking the derivative with respect to $P_{y}$ of the linear limit of $\Delta N\left(P_{y}\right)$, one finds

$$
\begin{aligned}
& \frac{\partial}{\partial P_{y}}\left(\lim _{P_{y} \rightarrow 0} \Delta N\left(P_{y}\right)\right) \\
& \quad=\frac{\partial}{\partial P_{y}}\left(\frac{\omega \epsilon_{0}}{2 k_{0}} \int_{-\infty}^{+\infty} n_{0}(x) n_{2 E} P_{y}\left|E_{n}(x)\right|^{4} d x\right) \\
& \quad=\frac{Q_{n}}{k_{0}}
\end{aligned}
$$

a result equal to (A.5). On the other hand, for the large power limit, one finds exactly the same result as (A.6). As in the case of an exponential nonlinearity, one can approximate the nonlinear effective index changes by a function with the same shape as the saturable nonlinearity itself. This leads to

$$
\Delta N(P)=\frac{\Delta N_{\text {sat }} \cdot \frac{P Q_{n}}{k_{0}}}{\Delta N_{\text {sat }}+\frac{P Q_{n}}{k_{0}}} .
$$

On comparing MSCF calculations with the results of (A.8) and (A.12), as in Fig. 3, it can be seen that the derived expressions give a very reasonable approximation of the saturation behavior of the effective index. Even in the case of a higher order mode (Fig. 3, lower graph), the induced effective index changes follow the curves as given by (A.8) and (A.12) despite the strong inhomogeneous saturation. This can be understood from (A.3) and (A.10); at positions where the intensity is small, the index changes are small and do not saturate, but at the same time, these index changes have relatively small weight in the integral too.

\section{B. Rough Estimate of Nonlinear Propagation Constants}

A rough, but simple estimate of the nonlinear effective indexes of guided modes of channel waveguides can be very helpful for easy order of magnitude calculations, and moreover, serve as a rough check of the correctness (of the implementation) of more elaborate methods. Such a simple expression is found when the field profiles are approximated by sines (for odd modes) and cosines (for even modes). For the lowest order $\mathrm{TE}_{00}$ mode, a suitable function is

$$
\begin{aligned}
& E_{00}(x, y)=\sqrt{\frac{P 8 \omega \mu_{0}}{\beta_{00} w_{\text {eff }}^{x} w_{\text {eff }}^{y}}} \cos \left(\frac{\pi x}{w_{\text {eff }}^{x}}\right) \cos \left(\frac{\pi y}{w_{\text {eff }}^{y}}\right) \\
& |x|<\frac{w_{\text {eff }}^{x}}{2} \forall|y|<\frac{w_{\text {eff }}^{y}}{2} \\
& E_{00}(x, y)=0 \quad|x|>\frac{w_{\text {eff }}^{x}}{2} \Lambda|y|>\frac{w_{\text {eff }}^{y}}{2} \quad \text { (A.13) }
\end{aligned}
$$

where $w_{\text {eff }}^{x}$ and $w_{\text {eff }}^{y}$ are the effective widths of the mode in the $x$ and $y$ direction, respectively [19]. The power carried by a mode as described by (A.13) is given by

$$
\frac{\beta_{00}}{2 \omega \mu_{0}} \int_{-\infty}^{+\infty} \int_{-\infty}^{+\infty} E_{00}^{2}(x, y) d x d y=P .
$$

When saturation is ignored, the induced nonlinear change in refractive index is given by

$$
\begin{aligned}
\Delta n(x, y) \approx & 2 n_{0} n_{2 E}\left|E_{00}(x, y)\right|^{2} \\
= & 2 n_{0} n_{2 E} \frac{P 8 \omega \mu_{0}}{\beta_{00} w_{\mathrm{eff}}^{x} w_{\mathrm{eff}}^{y}} \\
& \cdot \cos ^{2}\left(\frac{\pi x}{w_{\mathrm{eff}}^{x}}\right) \cos ^{2}\left(\frac{\pi x}{w_{\mathrm{eff}}^{x}}\right) .
\end{aligned}
$$

Application of the variation theorem [19] yields

$$
\begin{aligned}
& \frac{\Delta N_{00}}{P}=\frac{2 \omega \epsilon_{0} n_{0} n_{2 E}}{4 k_{0}}\left(\frac{8 \omega \mu_{0}}{\beta_{00} w_{\mathrm{eff}}^{x} w_{\mathrm{eff}}^{y}}\right)^{2} \\
& \quad \cdot \int_{-w_{\mathrm{eff}}^{x} / 2}^{+w_{\mathrm{eff}}^{x} / 2} \int_{-w_{\mathrm{eff}}^{y} / 2}^{+w_{\mathrm{eff}}^{y} / 2} \cos ^{4}\left(\frac{\pi x}{w_{\mathrm{eff}}^{x}}\right) \cos ^{4}\left(\frac{\pi x}{w_{\mathrm{eff}}^{y}}\right) d x d y .
\end{aligned}
$$

The integrals can be worked out independently for the $x$ and $y$ directions. Using the reduction formulas for the cosine twice the integrals yields a factor of $3 w_{\text {eff }}^{x} / 8 * 3 w_{\text {eff }}^{y} / 8$. Hence, the complete expression reads

$$
\frac{\Delta N_{00}}{P}=\frac{9 n_{0} n_{2 E}}{2 N_{\mathrm{eff}, 00}^{2} k_{0}} \frac{\omega \mu_{0}}{w_{\mathrm{eff}}^{x} w_{\mathrm{eff}}^{y}} .
$$


Expression (A.17) can also be obtained easily for higher order or odd modes. In order to do so, (A.13) and (A.15) have to be reevaluated. The results will have the same functional form as (A.17), but carry different proportionality constants which express the characteristics of the mode profiles involved.

\section{REFERENCES}

[1] A Kumar, D. Clarck, and B. Culshaw, "Explanations of errors inherent in the effective-index method for analyzing rectangularcore waveguides," Opt. Lett., vol. 13, pp. 1129-1131, 1988.

[2] F. Payne, "A new theory of rectangular optical waveguides," Opt. Quantum Electron., vol. 14, pp. 525-537, 1982.

[3] H. Hoekstra, "An economic method for the solution of the scalar wave equation for arbitrarily shaped optical waveguides," J. Lightwave Technol., vol. 8, pp. 789-793, 1990.

[4] K. Chiang, "Dual effective-index method for the analysis of rectangular waveguides," Appl. Opt., vol. 25, pp. 2169-2174, 1986

[5] C. Seaton, J. Valera, R. Shoemaker, G. Stegeman, J. Chilwell, and S. Smith, "Calculations of nonlinear TE waves guided by thin dielectric films bounded by nonlinear media," IEEE J. Quantum. Electron., vol. QE-21, pp. 774-783, 1985.

[6] A. Boardman, A. Maradudin, G. Stegeman, T. Twardowski, and E. Wright, "Exact theory of nonlinear p-polarized optical waves," Phys. Rev. A, vol. 35, pp. 1159-1164, 1987.

[7] W. Chen and A. Maradudin," $S$-polarized guided and surface electromagnetic waves supported by a nonlinear dielectric film," $J$. Opt. Soc. Amer. B, vol. 5, pp. 529-538, 1988.

[8] K. Hayata and M. Koshiba, "Self-focussing instability and chaotic behavior of nonlinear optical waves guided by dielectric slab structures," Opt. Lett., vol. 13, pp. 1041-1043, 1988.

[9] M. Ramadas, R.Varshney, K. Thyagarajan, and A. Ghatak, "A matrix approach to study the propagation characteristics of a general nonlinear planar waveguide," J. Lightwave Technol., vol. 7, pp. 1901-1905, 1989.

[10] J. Souza, "Numerical analysis and the route to chaos for $\mathrm{TE}_{1}$ waves in nonlinear slab waveguides," IEEE Photon. Technol. Lett., vol. 3, pp. 651-653, 1991.

[11] G. Stegeman and E. Wright, "All-optical waveguide switching," Opt. Quantum. Electron., vol. 22, pp. 95-121, 1990.

[12] Y. Silberberg and G. Stegeman, "Nonlinear coupling of waveguide modes," Appl. Phys. Lett., vol. 50, pp. 562-564, 1987.

[13] K. Hayata and M. Koshiba, "Full vectorial analysis of nonlinear optical waveguides," J. Opt. Soc. Amer. B, vol. 5, pp. 2494-2501, 1988.

[14] R. Ettinger, F. Fernandez, B. Rahman, and J. Davies, "Vector finite element solution of saturable nonlinear strip-loaded optical waveguides," IEEE Photon. Technol. Lett., vol. 3, pp. 147-149, 1991.

[15] N. N. Akhmediev, R. F. Nabiev, and Yu.M. Popov, "Three-dimensional modes of a symmetric nonlinear plane waveguide," Opt. Commun., vol. 69, pp. 247-252, 1989.

[16] _- "Stripe nonlinear waves in a symmetrical planar structure," Opt. Commun., vol. 72, pp. 190-194, 1989.

[17] Q. Y. Li, R. A. Sammut, and C. Pask, "Variational and finite element analysis of nonlinear strip optical waveguides," Opt. Commun., vol. 94, pp. 37-43, 1992.

[18] J. Chrostowski and S. Chelkowski, "Analysis of an optical rib waveguide with a nonlinear substrate," Opt. Lett., vol. 12, pp. 528-530, 1987.

[19] H. Kogelnik, "Theory of dielectric waveguides," in Integrated Op tics, Topics in Applied Physics 7, T. Tamir, Ed. Berlin, Heidelberg: Springer-Verlag, 1975.

[20] Q. Y. Li, C. Pask, and R. A. Sammut, "Simple model for spatial optical solitons in planar waveguides," Opt. Lett., vol. 16, pp. 1083-1085, 1991.

[21] R. A. Sammut, C. Pask, and Q. Y. Li, "Theoretical study of spatia solitons in planar waveguides," J. Opt. Soc. Amer. B, vol. $10 \mathrm{pp}$. 485-491, 1993.
[22] K. S. Chang and R. A. Sammut, "Effective index method for spatial solitons in planar waveguides with Kerr-type nonlinearity," J. Opt. Soc. Amer. B, vol. 10, pp. 704-708, 1993.

[23] F. Dios, L. Turner, and F. Canal, "Self-consistent solution for general nonlinear slab waveguides," Opt. Commun., vol. 72, pp. 54-59, 1989.

[24] G. Krijnen, "All-optical switching in nonlinear integrated optic devices," Ph.D. dissertation, Univ. Twente, Enschede, The Netherlands, May 1992, ISBN 90-9004929-0.

[25] R. Sammut and C. Pask, "Gaussian and equivalent-step-index approximations for nonlinear waveguides," J. Opt. Soc. Amer. B., vol. 8, pp. 395-402, 1991.

[26] See, for example, H. Hoekstra, G. Krijnen, P. Lambeck, and T. Popma, "Nonlinear optics for transducers: Principles and materials," in Digest of the Intermational Conference on Solid-State Sensors and Actuators, "Transducers '91," pp. 711-718 and references therein.

Gijs J. M. Krijnen (S'90-M'93) began his Ph.D. work in the Lightwave Devices Group of the MESA Research Institute at the University of Twente, Enschede, The Netherlands, in 1987, after receiving the M.Sc. degree following a master of sciences in magnetic recording at the Philips Laboratories, Eindhoven, The Netherlands. His Ph.D. research concerned integrated nonlinear optical switching devices.

After finishing his Ph.D. work in May 1992, he became a Postdoctoral Fellow at the Royal Netherlands Academy of Arts and Sciences. In this current work, his main interest is in the application of nonlinear integrated optics devices as sensors.

Hugo J. W. M. Hoekstra was born in 1949. He received the M.Sc. degree in experimental physics from the University of Amsterdam in 1977. After working as a teacher in high school, he began a Ph.D. study on magnetooptical properties of solid transition-metal halides at the University of Groningen where he received the $\mathrm{Ph} . \mathrm{D}$. degree in 1984.

Between 1984 and 1988 he was a Postdoctoral Fellow in the field of surface science at the Universities of Nijmegen and Groningen. In 1988 he joined the Lightwave Device Group of the MESA Research Institute at the University of Twente, Enschede, The Netherlands, where he is engaged in work on linear and nonlinear integrated optics.

Paul V. Lambeck was born in 1939. He received the M.Sc. degree in physical chemistry from the University of Amsterdam in 1964.

In the same year, he joined the University of Twente, Enschede, The Netherlands, starting in the field of ferroelectric materials, a study which resulted in his Ph.D. dissertation. Since 1984 he has been an Associate Professor in the Lightwave Device Group of the MESA Research Institute at the University of Twente, Enschede, The Netherlands. His current interest is in the field of integrated optics, and concentrates mainly on optical sensors and nonlinear devices. 